\title{
Bound States of Non-Hermitian Quantum Field Theories
}

\author{
Carl M. Bender ${ }^{1}$, Stefan Boettcher ${ }^{2}$, H. F. Jones ${ }^{3}$, Peter N. Meisinger ${ }^{1}$, and Mehmet \\ Șimșek ${ }^{10}$ \\ ${ }^{1}$ Department of Physics, Washington University, St. Louis, Missouri 63130, USA \\ ${ }^{2}$ Department of Physics, Emory University, Atlanta, Georgia 30322, USA \\ ${ }^{3}$ Blackett Laboratory, Imperial College, London SW7 2BZ, United Kingdom
}

(October 25, 2018)

\begin{abstract}
The spectrum of the Hermitian Hamiltonian $\frac{1}{2} p^{2}+\frac{1}{2} m^{2} x^{2}+g x^{4}(g>0)$, which describes the quantum anharmonic oscillator, is real and positive. The non-Hermitian quantum-mechanical Hamiltonian $H=\frac{1}{2} p^{2}+\frac{1}{2} m^{2} x^{2}-g x^{4}$, where the coupling constant $g$ is real and positive, is $\mathcal{P} \mathcal{T}$-symmetric. As a consequence, the spectrum of $H$ is known to be real and positive as well. Here, it is shown that there is a significant difference between these two theories: When $g$ is sufficiently small, the latter Hamiltonian exhibits a two-particle bound state while the former does not. The bound state persists in the corresponding non-Hermitian $\mathcal{P} \mathcal{T}$-symmetric $-g \phi^{4}$ quantum field theory for all dimensions $0 \leq D<3$ but is not present in the conventional Hermitian $g \phi^{4}$ field theory.
\end{abstract}

In this Letter we show that the spectrum of the non-Hermitian $\mathbb{\mathcal { P }} \mathcal{T}$-symmetric quartic Hamiltonian

\footnotetext{
${ }^{1}$ Contrary to appearances, this Hamiltonian is not Hermitian because its eigenfunctions are required to obey boundary conditions in the complex plane [see (田)].
} 


$$
H=\frac{1}{2} p^{2}+\frac{1}{2} m^{2} x^{2}-g x^{4} \quad(g>0)
$$

possesses bound states for sufficiently small $g$. Moreover, when the Euclidean space-time dimension $D$ satisfies $0 \leq D<3$, the corresponding $\mathcal{P} \mathcal{T}$-symmetric quantum field theory described by the Lagrangian

$$
\mathcal{L}=\frac{1}{2}(\nabla \phi)^{2}+\frac{1}{2} m^{2} \phi^{2}-g \phi^{4} \quad(g>0)
$$

also possesses bound states for small $g$. The related conventional Hermitian $g x^{4}$ and $g \phi^{4}$ theories with $g>0$ do not possess such bound states.

Quantum-mechanical Hamiltonians that are non-Hermitian but possess $\mathcal{P} \mathcal{T}$ symmetry have recently been studied in great detail by many authors [1-14. It is known that the entire spectrum of the $\mathcal{P} \mathcal{T}$-symmetric anharmonic oscillator is real and positive. The first proof of the reality of the spectrum is given in Ref. [13]. Direct numerical evidence for the reality and positivity of the spectrum can be found by performing a Runge-Kutta integration of the associated Schrödinger equation [1]. Alternatively, the large-energy eigenvalues of the spectrum can be calculated with great accuracy by using conventional WKB techniques [1.3, 15]. An easy way to demonstrate the reality and positivity of the spectrum is to calculate exactly the spectral zeta function (the sum of the inverses of the eigenvalues). This was done by Mezincescu and Bender and Wang [7, 9].

The calculations that are performed in this paper are perturbative; that is, quantities are determined as formal power series in the coupling constant $g$. Some explanation must be given to justify the application of perturbative methods to $\mathcal{P} \mathcal{T}$-symmetric theories. We will argue that to obtain a perturbation expansion for the theories described in (11) and (2) we need only calculate the perturbation series for the corresponding quantity in the conventional Hermitian $g x^{4}$ and $g \phi^{4}$ theories and change the sign of $g$ in these expansions. This procedure is correct but it is nontrivial, as we now argue.

First, consider the quantum-mechanical theory in (1). The corresponding differential equation for the energy eigenfunctions $\psi(x)$ is 


$$
-\frac{1}{2} \psi^{\prime \prime}(x)+\frac{1}{2} m^{2} x^{2} \psi(x)-g x^{4} \psi(x)=E \psi(x) \quad(g>0)
$$

where the wave function $\psi(x)$ satisfies boundary conditions in two wedges of $60^{\circ}$ opening angle in the complex- $x$ plane [1]:

$$
\lim _{|x| \rightarrow \infty} \psi(x)=0 \quad(-\pi / 3<\arg x<0 \quad \text { and } \quad-\pi<\arg x<-2 \pi / 3)
$$

These two wedges lie just below the real axis. Because they are symmetric with respect to the imaginary axis they enforce the $\mathcal{P} \mathcal{T}$ symmetry of the problem. In the limit as $g \rightarrow 0$, the differential equation in (3) becomes that of the harmonic oscillator and the boundary conditions are imposed in two wedges of $90^{\circ}$ opening angle in the complex- $x$ plane:

$$
\lim _{|x| \rightarrow \infty} \psi(x)=0 \quad\left(-\pi / 4<\arg x<\pi / 4 \quad \text { and } \quad-5 \pi / 4<\arg x<-\frac{3}{4} \pi\right) .
$$

Because the wedges for $g>0$ and the wedges for $g=0$ have a region in common, conventional perturbative methods are justified.

However, one must be extremely careful to avoid drawing wrong conclusions on the basis of perturbative calculations. For example, a perturbative calculation of the one-point Green's function (the expectation value of $x$ in the ground state) gives 0 to all orders in powers of the coupling constant $g$. Nevertheless, the correct result is nonzero, pure imaginary, and exponentially small [16]:

$$
G_{1} \equiv \frac{\int d x x \psi_{0}^{2}(x)}{\int d x \psi_{0}^{2}(x)} \sim-i K m^{3 / 2} g^{-2 / 3} e^{\frac{-4 m^{3}}{3 g}} \quad\left(g \rightarrow 0^{+}\right),
$$

where $K=8 \sqrt{\pi e} 3^{-1 / 6} / \Gamma^{2}(1 / 3)$ is a positive constant. The nonperturbative result in (6) is due to a soliton solution of the classical field equations.

There are even more subtle effects in which one may think that there are nonperturbative effects when there are none. For example, consider the cubic anharmonic oscillator Hamiltonian

$$
H=\frac{1}{2} p^{2}+\frac{1}{2} m^{2} x^{2}+g x^{3} \quad(g \text { real })
$$

A perturbative calculation of the ground-state energy has the form 


$$
E_{0} \sim \frac{1}{2} m+\sum_{1}^{\infty} a_{n}\left(g^{2}\right)^{n} \quad(g \rightarrow 0)
$$

The coefficients $a_{n}$ in this series are real and all have the same sign and thus the series is not Borel summable. The nonsummability of this series indicates the presence of a branch cut in the complex- $g$ plane on the real- $g$ axis. Thus, the function $E_{0}(g)$ is complex when $g$ is real. The imaginary part of $E_{0}(g)$ is a nonperturbative effect; it is exponentially small when $g$ is real and small. The energies of the cubic oscillator are complex because of tunneling effects on the real axis.

The corresponding $\mathcal{P} \mathcal{T}$-symmetric cubic oscillator, whose Hamiltonian is

$$
H=\frac{1}{2} p^{2}+\frac{1}{2} m^{2} x^{2}+i g x^{3} \quad(g \text { real })
$$

is obtained from the Hamiltonian in (耳) by replacing $g$ with $i g$. This replacement makes the series in (8) Borel summable [5,11] and verifies the result that eigenvalues of $H$ in (9) are all real.

However, this argument is quite tricky. Let us apply the same reasoning to the conventional Hermitian quantum-mechanical anharmonic oscillator described by

$$
H=\frac{1}{2} p^{2}+\frac{1}{2} m^{2} x^{2}+g x^{4} \quad(g>0)
$$

The Rayleigh-Schrödinger perturbation series for the ground-state energy of this Hamiltonian has the form

$$
E_{0} \sim \frac{1}{2} m+\sum_{1}^{\infty} a_{n} g^{n} \quad\left(g \rightarrow 0^{+}\right)
$$

where the coefficients $a_{n}$ are all real and alternate in sign. Hence, this series is Borel summable and we conclude (correctly) that the ground-state energy is real when $g>0$. We correctly obtain the perturbation expansion for the ground-state energy of the nonHermitian $\mathcal{P} \mathcal{T}$-symmetric anharmonic oscillator (1) by replacing $g$ with $-g$. Now, the coefficients in perturbation series for the ground-state energy no longer alternate in sign; the coefficients all have the same sign (they are all negative). However, all of the eigenvalues 
of the Hamiltonian (1) are real. Even though the series is not Borel summable, the imaginary part of the ground-state energy is exactly zero due to the presence of the soliton that leads to the nonperturbative result in (6).

The same discussion applies to the non-Hermitian $\mathcal{P} \mathcal{T}$-symmetric quantum field theory in (21). The perturbation expansions of the Green's functions of this theory are obtained using the same Feynman rules as for the conventional $g \phi^{4}$ quantum field theory, with the one change that the vertex amplitude $-24 g$ is replaced with $24 g$. However, again one must be very careful about nonperturbative effects. For example, the one-point Green's function, which vanishes in the conventional $g \phi^{4}$ quantum field theory, is now nonvanishing, pure imaginary, and exponentially small in the $-g \phi^{4}$ quantum field theory [16].

Having made these preliminary remarks, we turn to the question of bound states in $\mathcal{P} \mathcal{T}$ symmetric theories. Let us first examine a two-particle state for the conventional quantum anharmonic oscillator Hamiltonian (10). The small-g Rayleigh-Schrödinger perturbation series for the $k$ th energy level $E_{k}$ of this Hamiltonian is [17]

$$
E_{k} \sim m\left[k+\frac{1}{2}+\frac{3}{4}\left(2 k^{2}+2 k+1\right) \epsilon+\mathrm{O}\left(\epsilon^{2}\right)\right] \quad\left(\epsilon \rightarrow 0^{+}\right)
$$

where the dimensionless expansion parameter is $\epsilon=\mathrm{g} / \mathrm{m}^{3}$. In this theory the renormalized mass $M$ is defined as the first excitation above the ground state. Thus, from (12),

$$
M \equiv E_{1}-E_{0} \sim m\left[1+3 \epsilon+\mathrm{O}\left(\epsilon^{2}\right)\right] \quad\left(\epsilon \rightarrow 0^{+}\right)
$$

To determine if the two-particle state is bound, we examine the second excitation above the ground state using $(12)$. We define

$$
B_{2} \equiv E_{2}-E_{0} \sim m\left[2+9 \epsilon+\mathrm{O}\left(\epsilon^{2}\right)\right] \quad\left(\epsilon \rightarrow 0^{+}\right)
$$

If $B_{2}<2 M$, then a two-particle bound state exists and the binding energy, which is a negative quantity, is $B_{2}-2 M$. If $B_{2}>2 M$, then the second excitation above the vacuum is interpreted as an unbound two-particle state. We can see from (14) that in the smallcoupling region, where perturbation theory is valid, the conventional anharmonic oscillator 
does not possess a bound state. Indeed, using a variety of methods (WKB theory, variational methods, numerical calculations) one can show that there is no two-particle bound state for any value of $g>0$. Because there is no bound state the $g x^{4}$ interaction may be considered to represent a repulsive force. 2

We obtain the perturbation series for the $\mathcal{P} \mathcal{T}$-symmetric oscillator, whose Hamiltonian $H$ is given in (1), from the perturbation series for the conventional anharmonic oscillator by replacing $\epsilon$ with $-\epsilon$. Thus, while the conventional anharmonic oscillator does not possess a two-particle bound state, evidently the $\mathcal{P} \mathcal{T}$-symmetric oscillator does indeed possess such a state. The binding energy of this state can be measured in units of the renormalized mass $M$. For this purpose we define the dimensionless binding energy $\Delta_{2}$ by

$$
\Delta_{2} \equiv \frac{B_{2}-2 M}{M} \sim-3 \epsilon+\mathrm{O}\left(\epsilon^{2}\right) \quad\left(\epsilon \rightarrow 0^{+}\right)
$$

In Fig. 1, $\Delta_{2}$ is plotted as a function of the dimensionless coupling constant $\epsilon$. Observe that the bound state disappears when the value of $\epsilon$ increases beyond $\epsilon=0.0465 \ldots$. As $\epsilon$ continues to increase, $\Delta_{2}$ reaches a maximum value of 0.427 at $\epsilon=0.13$ and then approaches the limiting value 0.28 as $\epsilon \rightarrow \infty$.

It is interesting that the bound state for the $\mathcal{P} \mathcal{T}$-symmetric anharmonic oscillator occurs only for values of $\epsilon$ less than about 0.0465 . The following heuristic argument helps to explain why the characteristic size of $\epsilon$ is so small. For the Hamiltonian in (1) the potential has the form $V(x)=\frac{1}{2} m^{2} x^{2}-g x^{4}$. On the real- $x$ axis, the maximum value of $V(x)$ occurs at $x^{2}=\frac{1}{4} m^{2} / g$ and at this point $V_{\max }=\frac{1}{16} m^{4} / g$. In order to have a two-particle bound state

\footnotetext{
${ }^{2}$ In general, a repulsive force in a quantum field theory is represented by an energy dependence in which the energy of a two-particle state decreases with separation. The conventional anharmonic oscillator Hamiltonian corresponds to a field theory in one space-time dimension, where there cannot be any spatial dependence. In this case the repulsive nature of the force is understood to mean that the energy $B_{2}$ needed to create two particles at a given time is more than twice the energy $M$ needed to create one particle.
} 
it is necessary for the height of the potential to be high enough to bind three states, the largest of which is of order $\frac{5}{2} \mathrm{~m}$. Setting this value equal to $V_{\max }$ gives $\epsilon=\frac{1}{40}$, which is near the value for which the two-particle state is most strongly bound. Note that the potential $V(x)$ does not allow states to tunnel out of the well, even though the potential decreases for large real $x$. This is because the $\mathcal{P} \mathcal{T}$-symmetric boundary conditions are not imposed on the real axis but rather in a pair of wedges in the complex- $x$ plane that lie below the real axis [see Eq. (四)].

In the $\mathcal{P} \mathcal{T}$-symmetric anharmonic oscillator, there are not only two-particle bound states for small coupling constant but also $k$-particle bound states for all $k \geq 2$. To see why, we calculate the energy of the $k$ th excitation above the vacuum:

$$
B_{k} \equiv E_{k}-E_{0} \sim m\left[k-\frac{3}{2}\left(k^{2}+k\right) \epsilon+\mathrm{O}\left(\epsilon^{2}\right)\right] \quad(\epsilon \rightarrow 0+)
$$

which is the generalization of (14). Thus, the dimensionless binding energy is

$$
\Delta_{k} \equiv \frac{B_{k}-k M}{M} \sim-\frac{3}{2} k(k-1) \epsilon+\mathrm{O}\left(\epsilon^{2}\right) \quad(\epsilon \rightarrow 0+)
$$

This equation reduces to (15) when $k=2$. The key feature of this equation is that the coefficient of $\epsilon$ is negative. Since the dimensionless binding energy becomes negative as $\epsilon$ increases from 0 , there is a $k$-particle bound state. The dimensionless binding energies for the first five $k$-particle bound states are shown in Fig. 2. Note that the higher multiparticle bound states cease to be bound for smaller values of $\epsilon$; starting with the three-particle bound state, the binding energy of these states becomes positive as $\epsilon$ increases past 0.039, 0.034, 0.030, and 0.027. Furthermore, the heuristic argument given above for the value of $\epsilon$ at which the multiparticle states are most strongly bound is qualitatively correct; one can see that the $k$-particle bound state is most strongly bound at a value of $\epsilon$ that is roughly $1 /(16 k+8)$.

Figure 2 shows that for any value of $\epsilon=\mathrm{g} / \mathrm{m}^{3}$ there are always a finite number of bound states and an infinite number of unbound states. The number of bound states decreases with increasing $\epsilon$ until, as we see in Fig. 1, there are no bound states at all. Observe that 
there is a range of $\epsilon$ for which there are only two- and three-particle bound states. (This situation is analogous to the physical world in which one observes only states of two and three bound quarks.) In this range of $\epsilon$ if one has an initial state containing a number of particles (renormalized masses), these particles will clump together into bound states, releasing energy in the process. Depending on the value of $\epsilon$, the final state will consist either of two- or of three-particle bound states, whichever is energetically favored. Note also that there is a special value of $\epsilon$ for which two- and three-particle bound states can exist in thermodynamic equilibrium.

Let us now generalize the result for the quantum-mechanical two-particle bound state in (15) to the case of the $D$-dimensional $\mathcal{P} \mathcal{T}$-symmetric quantum field theory in (2). To show that there is a bound state, we need only demonstrate that to leading order in the coupling constant, the binding energy becomes negative as the coupling constant increases from 0. The Feynman rules for the calculation are the conventional ones, except that the sign of the vertex is reversed: $24 g$ for a four-point vertex amplitude and $1 /\left(p^{2}+M^{2}\right)$ for a line amplitude. Note that to leading order in the coupling constant we use the renormalized mass $M$ rather than the unrenormalized mass $m$ in the propagator amplitude and we exclude self-energy loops in the graphs.

To calculate the energy of the bound state to leading order we sum up all "sausage-link" graphs and identify the bound-state pole in the geometric series. These graphs are shown in Fig. 3. The result for the dimensionless binding energy to leading order in the dimensionless coupling constant $\epsilon=g M^{D-4}$ is

$$
\Delta_{2} \sim-4\left[\frac{3 \Gamma\left(\frac{3-D}{2}\right)}{4 \pi^{(D-1) / 2}}\right]^{2 /(3-D)} \epsilon^{2 /(3-D)},
$$

where $D$ is the Euclidean space dimension. The formula reduces to that in (15) for the case $D=1$. This formula is valid for $0 \leq D<3$. Note that we have performed a mass renormalization to leading order, but we have not done a wave function or coupling-constant renormalization in this calculation.

We conclude by remarking that the behavior of a $g \phi^{3}$ theory is the reverse of that of a 
$g \phi^{4}$ theory. A $g \phi^{3}$ theory represents an attractive force. The bound states that arise as a consequence of this force can be found by using the Bethe-Salpeter equation. However, the $g \phi^{3}$ field theory is unacceptable because the spectrum is not bounded below. If we replace $g$ by $i g$, the spectrum now becomes real and positive, but the force becomes repulsive and there are no bound states. The same is true for a two-scalar theory with interaction of the form $i g \phi^{2} \chi$. This latter theory is an acceptable model of scalar electrodynamics, but has no analog of positronium.

\section{ACKNOWLEDGMENTS}

MṢ is grateful to the Physics Department at Washington University for their hospitality during his sabbatical. This work was supported by the U.S. Department of Energy. 


\section{REFERENCES}

* Permanent address: Gazi Universitesi, Fen Edebiyat Fakultesi, Fizik Bolumu, 06500 Teknikokullar-Ankara, Turkey.

[1] C. M. Bender and S. Boettcher, Phys. Rev. Lett. 80, 5243 (1998).

[2] E. Delabaere and F. Pham, Phys. Lett. A 250, 25 (1998) and 29 (1998).

[3] C. M. Bender, S. Boettcher, and P. N. Meisinger, J. Math. Phys. 40, 2201 (1999).

[4] C. M. Bender, F. Cooper, P. N. Meisinger, and V. M. Savage, Phys. Lett. A 259, 224 (1999).

[5] C. M. Bender and G. V. Dunne, J. Math. Phys. 40, 4616 (1999).

[6] E. Delabaere and D. T. Trinh, J. Phys. A: Math. Gen. 33, 8771 (2000).

[7] G. A. Mezincescu, J. Phys. A: Math. Gen. 33, 4911 (2000).

[8] C. M. Bender, S. Boettcher, and V. M. Savage, J. Math. Phys. 41, 6381 (2000).

[9] C. M. Bender and Q. Wang, to be published in J. Phys. A: Math. Gen.

[10] K. C. Shin, University of Illinois preprint.

[11] C. M. Bender and E. J. Weniger, J. Math. Phys. 42, 2167-2183 (2001).

[12] C. M. Bender, G. V. Dunne, P. N. Meisinger, and M. Șimṣek, Phys. Lett. A 281, 311-316 (2001).

[13] P. Dorey, C. Dunning, and R. Tateo, hep-th/0103051.

[14] C. M. Bender, M. Berry, P. N. Meisinger, V. M. Savage, and M. Șimṣek, J. Phys. A: Math. Gen. 34, L31-L36 (2001).

[15] C. M. Bender and S. A. Orszag, Advanced Mathematical Methods for Scientists and Engineers (McGraw-Hill, New York, 1978), Chap. 10. 
[16] C. M. Bender, P. Meisinger, and H. Yang, Phys. Rev. D 63, 45001 (2001).

[17] C. M. Bender and T. T. Wu, Phys. Rev. D 7, 1620 (1973), Eq. (5.11). 


\section{FIGURES}

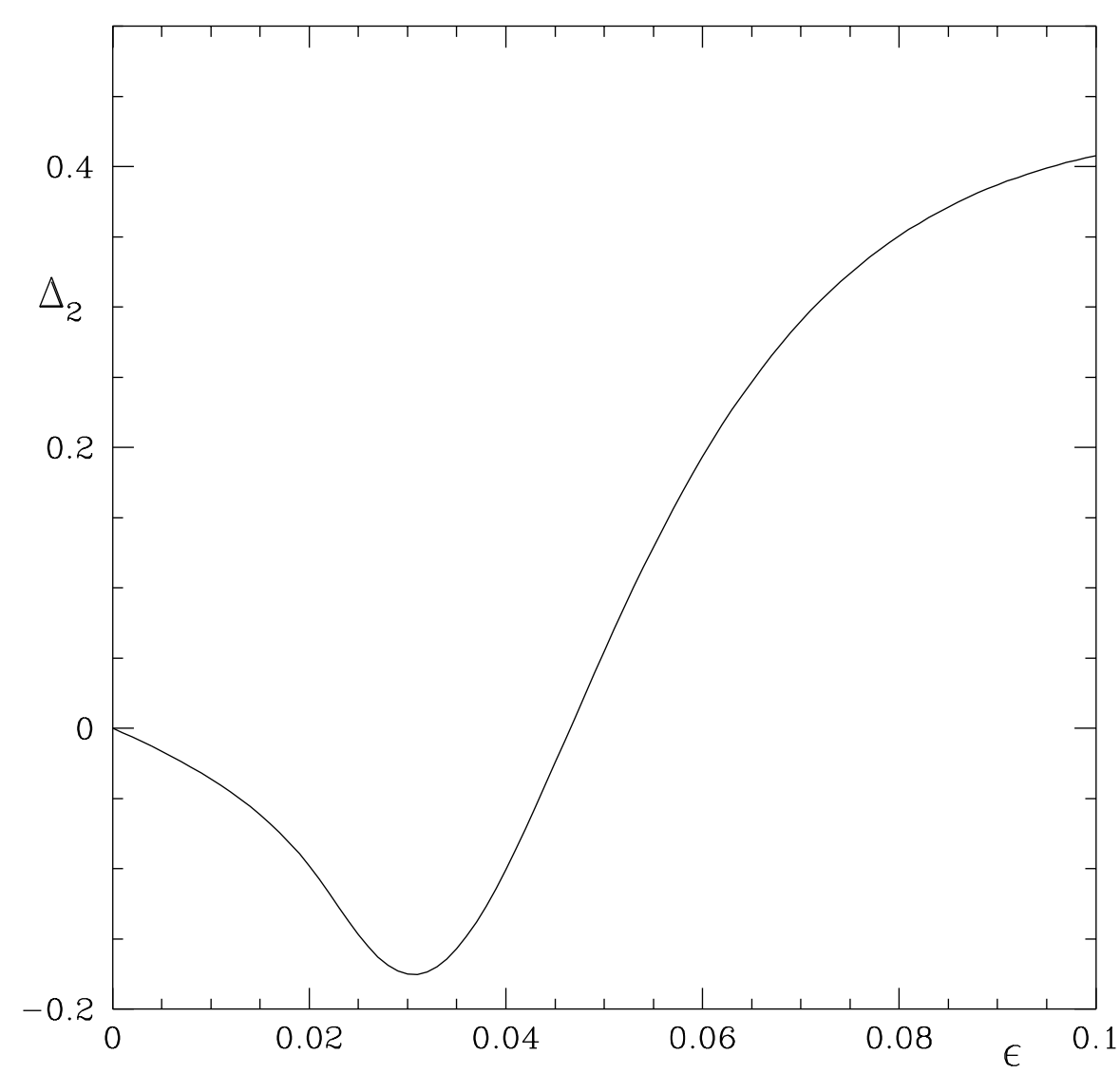

FIG. 1. Dimensionless binding energy $\Delta_{2}$ of the two-particle bound state of the $\mathcal{P} \mathcal{T}$-symmetric anharmonic oscillator, whose Hamiltonian $H$ is given in Eq. (1), plotted as a function of the dimensionless coupling constant $\epsilon=\mathrm{g} / \mathrm{m}^{3}$. The quantity $\Delta_{2}$ represents the binding energy $B_{2}$ measured in units of the renormalized mass [see Eq. (15)]. Note that for small $\epsilon$ the slope of the curve is -3 , which verifies the asymptotic result in (15). Observe that the bound state disappears when $\epsilon$ increases beyond $0.0465 \ldots$. As $\epsilon$ continues to increase, $\Delta_{2}$ reaches a maximum value of 0.427 at $\epsilon=0.13$. Then $\Delta_{2}$ decreases and approaches the limiting value 0.28 as $\epsilon \rightarrow \infty$. 


\section{FIGURE 2}

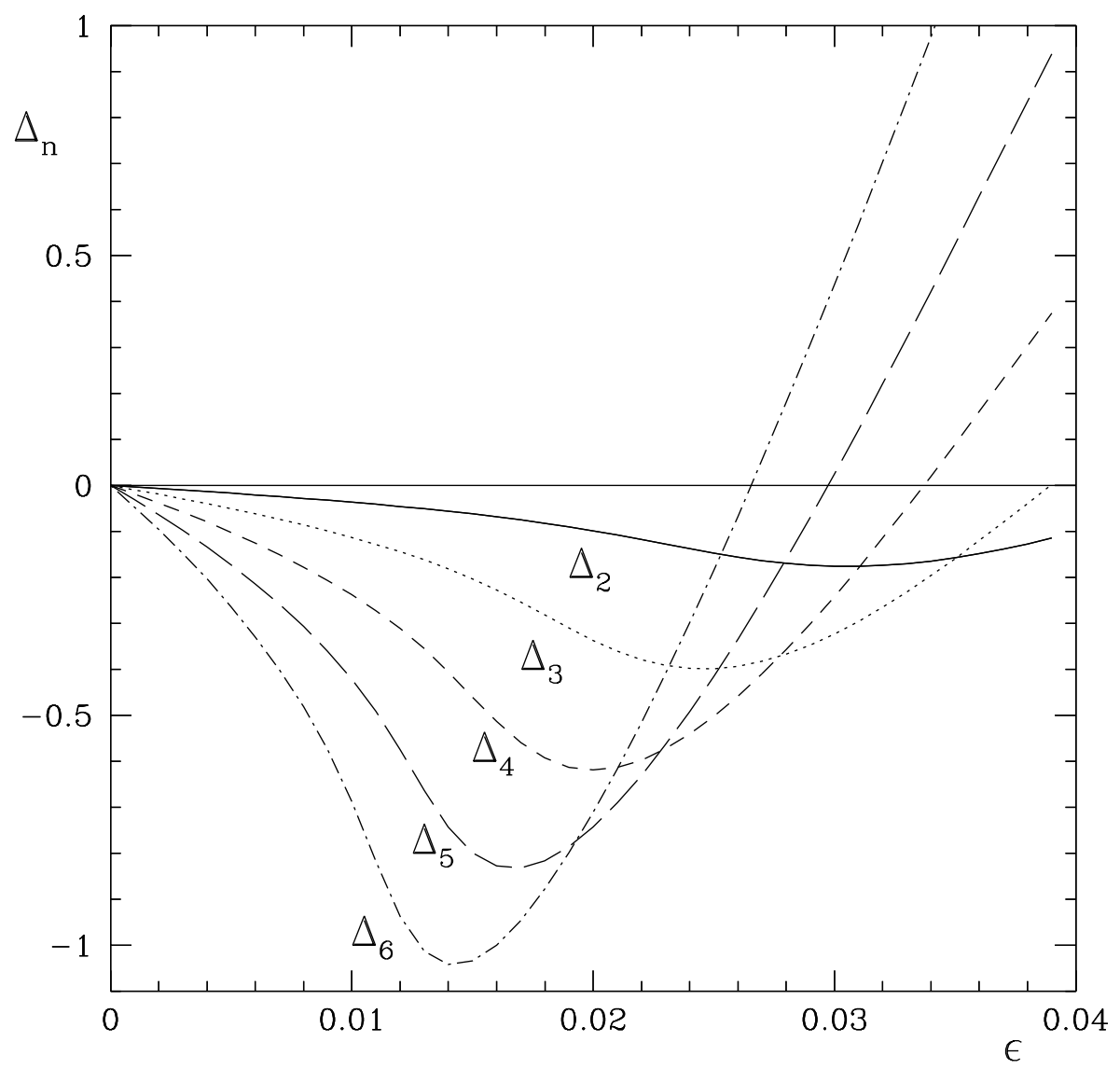

FIG. 2. Dimensionless binding energies $\Delta_{2}, \Delta_{3}, \Delta_{4}, \Delta_{5}$, and $\Delta_{6}$ for the two-, three-, four-, five-, and six-particle bound states plotted as functions of the dimensionless coupling constant $\epsilon$. Note that the multiparticle bound states cease to be bound as $\epsilon$ increases past $0.039,0.034,0.030$, and 0.027 . 


\section{FIGURE 3}

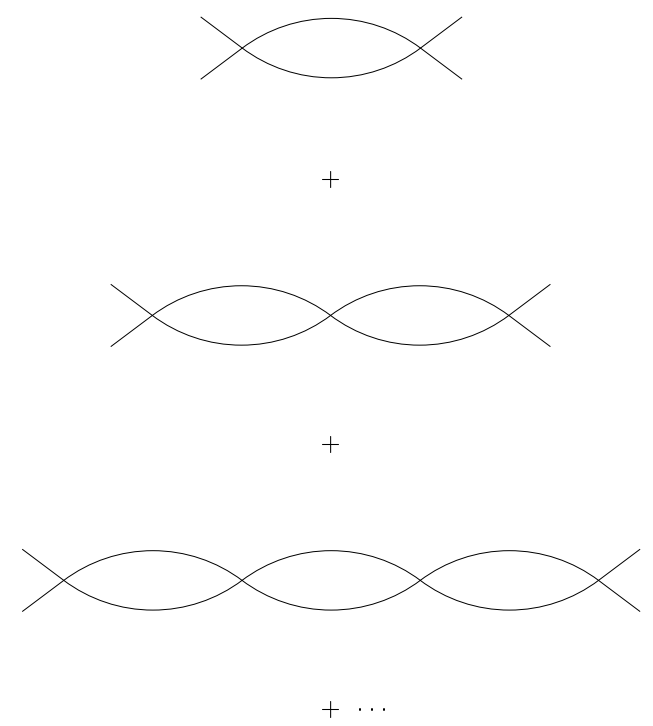

FIG. 3. So called "sausage-link" graphs contributing to the leading-order value of the energy of the two-particle bound state for the $\mathcal{P} \mathcal{T}$-symmetric $-g \phi^{4}$ quantum field theory whose Euclidean Lagrangian is given in Eq. (2). 